\title{
Optimizing the Roles of Unit and Non-unit Protection Methods Within DC Microgrids
}

\author{
Steven D. A. Fletcher, Student Member, IEEE, Patrick J. Norman, Stuart J. Galloway, Paul Crolla, Member, IEEE, \\ and Graeme M. Burt, Member, IEEE
}

\begin{abstract}
The characteristic behavior of physically compact, multiterminal de networks under electrical fault conditions can produce demanding protection requirements. This represents a significant barrier to more widespread adoption of de power distribution for microgrid applications. Protection schemes have been proposed within literature for such networks based around the use of non-unit protection methods. This paper shows however that there are severe limitations to the effectiveness of such schemes when employed for more complex microgrid network architectures. Even current differential schemes, which offer a more effective, though costly, protection solution, must be carefully designed to meet the design requirements resulting from the unique fault characteristics of dc microgrids. This paper presents a detailed analysis of dc microgrid behavior under fault conditions, illustrating the challenging protection requirements and demonstrating the shortcomings of non-unit approaches for these applications. Whilst the performance requirements for the effective operation of differential schemes in dc microgrids are shown to be stringent, the authors show how these may be met using COTS technologies. The culmination of this work is the proposal of a flexible protection scheme design framework for dc microgrid applications which enables the required levels of fault discrimination to be achieved whilst minimizing the associated installation costs.
\end{abstract}

Index Terms-DC power systems, fault currents, microgrid, power system protection.

\section{INTRODUCTION}

W ITHIN microgrid systems, dc power distribution has the potential to offer more efficient interconnection of distributed energy resources, such as small-scale generation, backup energy storage, and some industrial and sensitive electronic loads [1], [2]. Using de distribution it is often possible to reduce the number of power conversion stages for both the connection of distributed sources and loads, which can increase overall system efficiency [2], [3]. The paralleling of multiple sources onto a dc bus is more straight forward than for an ac bus, as the requirement for tight frequency regulation of the supply is removed [4]. This enables the faster connection of sources

Manuscript received July 29, 2011; revised December 21, 2011; accepted April 11, 2012. This work was supported by the U.K. Engineering and Physical Sciences Research Council. Paper no. TSG-00277-2011.

The authors are with the Institute for Energy and Environment, Department of Electronic and Electrical Engineering, University of Strathclyde, Glasgow G11XW, U.K. (e-mail: sfletcher@eee.strath.ac.uk; pnorman@eee.strath.ac.uk; stuart.galloway@eee.strath.ac.uk; paul.crolla@eee.strath.ac.uk; g.burt@eee. strath.ac.uk).

Color versions of one or more of the figures in this paper are available online at http://ieeexplore.ieee.org.

Digital Object Identifier 10.1109/TSG.2012.2198499 to the grid, providing better dynamic performance and allowing greater use of renewable sources under intermittent conditions. It is also possible to transmit more dc power through a cable of a given voltage rating than with ac due to the higher average voltage level, which could facilitate a reduction in cable sizes (with associated cost savings) [5]. Additionally, dc systems are inherently free from skin effect and reactive voltage drop, further improving power transfer.

However, the lack of effective solutions to date for electrical fault protection of dc systems represents a significant barrier to more widespread adoption of dc. The inherent challenges associated with dc include protecting against currents with high magnitude and rate of change in a fast but coordinated way, the prevention of significant voltage transients when operating protection, and the design of dc circuit breaker technologies to operate at required speed, voltage and current levels [1], [6]. As a result of these issues, there has been considerable attention in recent years on developing novel protection systems for dc applications.

One particular aspect often overlooked in the protection of dc microgrid networks is the high sensitivity of the network response to fault impedance. This is particularly evident from recent research which proposes the use of a protection scheme based on non-unit protection techniques without full consideration of fault impedance [4], [7]. Implementation of such a scheme on more complex dc microgrid architectures may lead to suboptimal fault discrimination (that is, ensuring that only the local protection operates for a fault at a particular location in the network) resulting in either longer fault clearance times or the disconnection of larger parts of a network than necessary in the event of a fault. This will be demonstrated in later sections of this paper.

To achieve greater levels of fault discrimination within these networks, the implementation of a unit protection scheme can be necessary. However, the scope for the implementation of unit protection is typically limited within distribution systems due to the additional cost of the necessary communication and relay technology. On the other hand, the development of smart grid and microgrid concepts will lead to an increase in the amount of sensor and communication infrastructure within distribution and low voltage networks [8], [9]. The purpose of this enhancement in infrastructure is to enable advanced automatic network monitoring and management algorithms to be embedded within the system, to increase usage of intermittent sources and decrease network congestion. The deployment of this advanced infrastructure also provides the opportunity to expand the use of unit protection schemes within microgrids. 


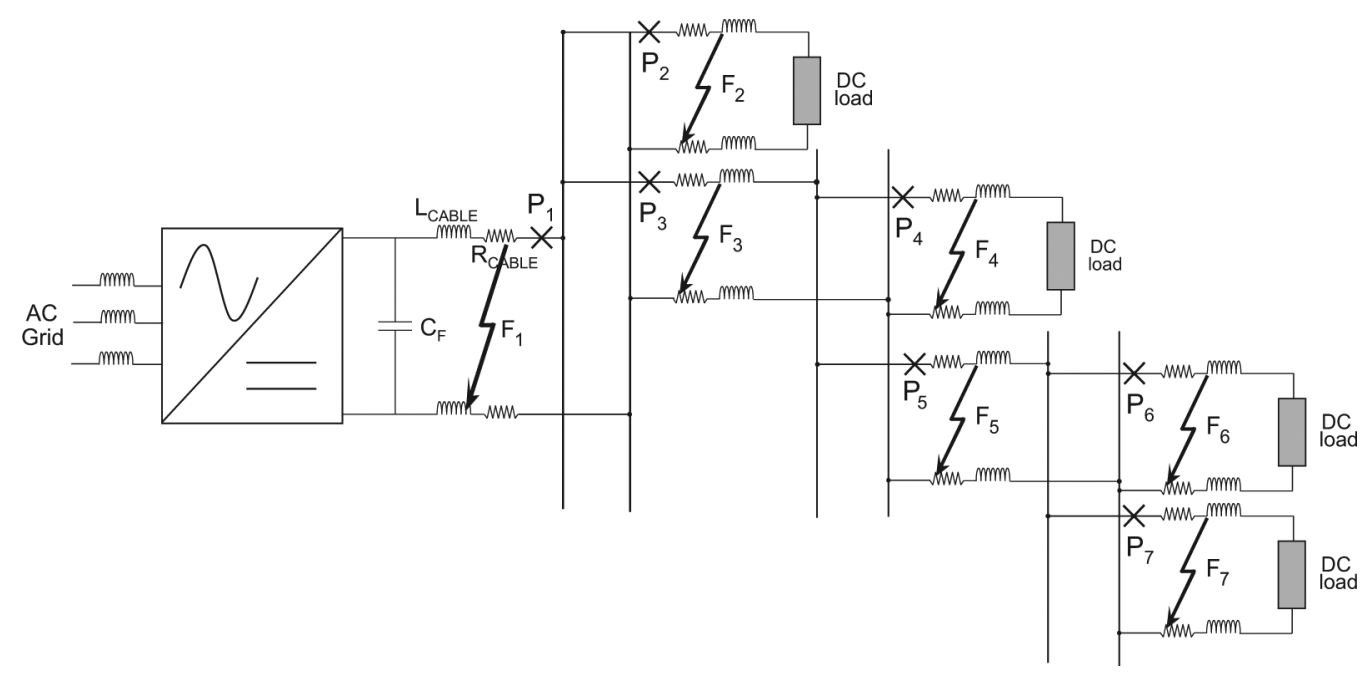

Fig. 1. Network diagram.

This paper first investigates the capability of non-unit protection methods to achieve effective protection discrimination within dc microgrid systems by analytically describing the current, voltage, $d i / d t, d v / d t$ and impedance response for a range of fault locations and impedances. The particular difficulties of achieving fast acting and discriminative protection using nonunit methods are subsequently demonstrated using an analytical case study. Differential current behavior within dc microgrids is then analyzed, facilitating the identification of, and the proposal of solutions for, the challenging measurement and signal synchronization requirements for unit schemes. Finally, a protection system design framework for dc microgrid applications is proposed which provides optimal protection discrimination and operating speed whilst minimizing system installation costs.

\section{Quantification of DC Protection System OPERATING REQUIREMENTS}

Cuzner [1] outline the key design criteria for any protection system and these relate to the operability and cost of a protection system. From the criteria presented, the performance of the protection system is assessed on its ability to provide continuity of supply to loads where other parts of the network are experiencing faults.

The dc microgrid network used as the basis for comparison within this paper is presented within Fig. 1, with Table I presenting relevant network parameters. This network has been derived from example architectures within literature [4], [7] and is supplied by a voltage source converter (VSC). Only a single source has been considered within Fig. 1 to simplify analysis and illustration however it is anticipated that findings will be applicable to networks with multiple sources. To ensure minimum disruption to the network presented within Fig. 1 in the event of a fault, protection devices $P_{1}$ to $P_{7}$ must operate in a coordinated way, such that only the device immediately upstream from the fault operates.

However there are a number of factors which influence the time-frame within which the network protection has to coordinate its devices operation. Many of these factors center around the use of use of a VSC as the main network supply.
TABLE I

NETWORK PARAMETERS

\begin{tabular}{llllll}
\hline Voltage $(\mathrm{V})$ & $P_{G E N}(\mathrm{~kW})$ & $R_{C A B L E}(\mathrm{~m} \Omega / \mathrm{m})$ & $L_{C A B L E}(\mu \mathrm{H} / \mathrm{m})$ & $C_{F}(\mathrm{mF})$ & $C_{E S R}(m \Omega)$ \\
\hline 400 & 320 & 0.641 & 0.65 & 56 & 2 \\
\hline
\end{tabular}

Previous work has highlighted that the fast discharge of capacitors used as filters on the de terminals of the VSC can damage both the capacitors themselves and any other sensitive components in the fault path [7]. Considerable short term electromagnetic forces on conductors can also be induced, creating risks of physical damage to mountings or insulation [10]. Furthermore, [6] and [11] illustrate the potential for voltage reversal if dc side faults are not cleared within an adequate time frame. The voltage reversal can cause significant currents to flow through converter freewheeling diodes, causing damage to these devices.

The fault current withstand of VSCs is low compared to more robust thyristor based converter topologies [4], [7], therefore current must be limited or interrupted much more quickly to prevent damage to internal components when supplying fault current.

The typical topology of VSC devices is such if the back-biasing dc voltage is lost after the occurrence of a fault, the antiparallel diodes across the switching devices will begin to conduct, meaning the converter is unable to block the flow of current to the fault [6], [12]. For these converter types, it necessary for network protection to act quickly to prevent damaging currents from flowing through the diodes, within $2 \mathrm{~ms}$ in some cases [4].

Alternative VSC topologies contain their own internal protection functionality, which enables the interruption of current flow through the converter. An example topology capable of this is provided within [7], where antiparallel diodes are replaced with emitter turn-off devices. Internal converter protection can be sensitive to overcurrent, overvoltage or undervoltage [13], [14]; however as the only source of fault current within Fig. 1 
TABLE II

REQUiRED TRIPPING TIMES FOR UNDERVOLTAGE THRESHOLD OF 200 V FOR A 1 -m $\Omega$ Fault at Various Fault Locations

\begin{tabular}{ll}
\hline Fault Location & $\begin{array}{l}\text { Time to undervoltage } \\
\text { threshold after fault }(m s)\end{array}$ \\
\hline$F_{1}$ & 0.9 \\
\hline$F_{2}$ & 2.2 \\
\hline$F_{3}$ & 2.2 \\
\hline$F_{4}$ & 3.7 \\
\hline$F_{5}$ & 3.7 \\
\hline$F_{6}$ & 5.3 \\
\hline
\end{tabular}

it is essential that the converter protection coordinates with protection devices $P_{1}$ to $P_{7}$ to ensure that only the appropriate protection device operates prior to converter protection operation.

Operational standards do exist for ac and HVDC systems which describe the requirements for converter connection in the event of network fault conditions. For example, [14] stipulates that in the event of a network undervoltage, converters are required to remain connected for a minimum of $140 \mathrm{~ms}$ to avoid sympathetic tripping for faults elsewhere in the network [15]. However it is difficult to see how these requirements apply to less robust converter types, where connection for this period of time may result in the flow of damaging current magnitudes flowing through the converter.

Whilst converter undervoltage protection is typically not as important as overcurrent for preventing device damage, for a $\mathrm{dc}$ system the undervoltage is a consequence of filter capacitor discharge, which in itself may cause problems. An undervoltage will be followed by an overcurrent condition on the ac side of the converter, as more current is drawn to attempt to recover the dc voltage. The dc side undervoltage can also be linked to the operation of ac side protection, which may monitor both dc voltage and current to determine its operation [13].

Given that the de voltage is linked to a number of aspects of the network and converter protection, it is useful to consider the voltage response when deriving protection system operating criteria. The dc voltage response has the added advantage of being least dependent on ac network conditions and configuration, and hence provides a dc side solution which could be deployed within multiple applications. For these reasons, this paper assesses the potential for current fault detection methods to coordinate with a converter undervoltage threshold for the network described within Fig. 1.

To derive a fixed operating point, an undervoltage threshold of $200 \mathrm{~V}$ (half the nominal system voltage) has been selected. It should however be noted that the observations in the following sections are relevant for various voltage thresholds. Table II highlights the time at which this voltage threshold is reached following the occurrence of a 1-m $\Omega$ fault at the six fault locations indicated in Fig. 1.

From Table II it is clear that, for the range of low impedance faults considered, the rapid loss of voltage at the converter terminals creates particularly challenging times for the operation of protection if it is to act to prevent the undervoltage occurring. The times identified are much shorter than required for ac converter connection [14], although they are in fact similar in magnitude to the requirements derived in [4] for prevention of overcurrent, highlighting the unique challenges for the type of network considered.

The following sections will demonstrate the challenges in achieving discriminatory protection system operation within the time frames outlined using of non-unit methods.

\section{Analysis of Non-unit Protection Method EFFECTIVENESS}

Non-unit protection does not protect a clearly bounded zone of the power system and will operate whenever its threshold is violated; non-unit schemes have inherent backup capabilities and will act to protect the system if a neighboring protection system fails to operate [16]. Recent research [4], [7] outlines approaches for the use of non-unit based protection scheme methods on microgrid and other multiterminal dc applications. Whilst non-unit approaches proved effective for the application considered, the architecture of these networks was such that there was not the requirement to coordinate upstream and downstream devices in any significant way.

This section investigates the capability of non-unit protection methods to achieve effective protection selectivity, within the times derived within the previous section, for the more complex dc system illustrated within Fig. 1. The section first describes the current, voltage, $d i / d t, d v / d t$, and impedance profiles as measured at the generator converter terminals to enable a better understanding of the network fault response. To enable this analysis to be more easily understood and usefully employed, simplifying assumptions have been made, which are based on previous work by the authors and presented in [6]. The key findings from this analysis can then be considered for specific protection schemes and an example case study, based on an overcurrent scheme, is presented to illustrate the challenges faced.

\section{A. Analysis of Dc Microgrid Fault Response}

Under fault conditions, the initial current response of the network generally takes one of two forms depending on circuit damping conditions [6]. For an underdamped circuit (defined below), current can be defined as

$$
i(t)=\frac{v_{C F}(0)}{L \omega_{d}} e^{-\alpha t} \sin \left(\omega_{d} t\right)
$$

where $v_{C F}(0)$ is the initial voltage on filter capacitance $C_{F}$. In (1), the term $\omega_{d}$ represents the damped resonant frequency and is defined as

$$
\omega_{d}=\sqrt{\omega_{0}^{2}-\alpha^{2}}
$$

$\alpha$ is the damping factor (or Neper frequency) and is defined as

$$
\alpha=\frac{R}{2 L}
$$

the term $\omega_{0}$ is the resonant radian frequency and is defined as

$$
\omega_{0}=\frac{1}{\sqrt{L C_{F}}}
$$

and $R$ and $L$ are the total resistance and inductance between the discharging capacitor and the fault. Note that (1) assumes that initial current (at $t=0$ ) in the circuit is zero as the dominant 
part the fault current characteristic is usually due to the initial voltage across the converter filter capacitance [6].

The damping conditions in the network depend on the terms $\omega_{0}$ and $\alpha$. The circuit is considered underdamped if $\omega_{0}^{2}>\alpha^{2}$, and overdamped if $\alpha^{2}>\omega_{0}^{2}$. For the overdamped case, current is defined as

$$
i(t)=\frac{v_{C F}(0)}{L\left(s_{1}-s_{2}\right)}\left(e^{s_{1} t}-e^{s_{2} t}\right)
$$

again neglecting initial current, and where

$$
s_{1,2}=-\alpha \pm \sqrt{\alpha^{2}-\omega_{0}^{2}}
$$

Current magnitude is often not used in itself to discriminate fault location but is also coupled with time, with a common form being based on the $i^{2} t$ response of the network. The $i^{2} t$ expressions for the two damping conditions, neglecting initial current, can be derived by squaring and integrating (1) and (5), respectively. These are

$$
\begin{aligned}
i^{2} t=\frac{v_{C F}(0)^{2} C}{L} & {\left[-\frac{1}{4 \alpha}-\frac{\alpha}{2 \omega_{d}^{2}}+e^{-2 \alpha t}\right.} \\
& \left(-t+\frac{1}{4}\left[\frac{1}{\alpha}+\frac{\alpha}{\omega_{d}^{2}}\left(1+\cos \left(2 \omega_{d} t\right)\right.\right.\right. \\
& \left.\left.\left.\left.-\frac{\sin \left(2 \omega_{d} t\right)}{\omega_{d}}\right)\right]\right)\right]
\end{aligned}
$$

for underdamped conditions and

$$
\begin{aligned}
i^{2} t=\frac{v_{C F}(0)^{2}}{L^{2}\left(s_{1}-s_{2}\right)^{2}} & {\left[\frac{1}{2 s_{1}}\left(1+e^{2 s_{1} t}\right)\right.} \\
& \left.-\frac{2}{s_{1} s_{2}}\left(1+e^{s_{1} s_{2} t}\right)-\frac{1}{2 s_{2}}\left(1+e^{2 s_{2} t}\right)\right]
\end{aligned}
$$

for overdamped network conditions.

Whilst it is more common to use current to detect faults, alternative measures such as voltage [17], $d i / d t$ [18], $d v / d t$ [19], and impedance [20] can be used for fault detection and are therefore worthy of consideration. Conveniently, these can be derived from the current response, as described below.

The voltage response is proportional to the integral of current and, over the faulted period, can be represented by

$$
v(t)=v_{C F}(0) e^{-\alpha t}\left[\cos \left(\omega_{d} t\right)+\frac{\alpha}{\omega_{d}} \sin \left(\omega_{d} t\right)\right]
$$

for underdamped conditions and

$$
v=\frac{v_{C F}(0) \omega_{0}^{2}}{\left(s_{1}-s_{2}\right)}\left(\frac{e^{s_{1} t}}{s_{1}}-\frac{e^{s_{2} t}}{s_{2}}\right)
$$

for overdamped conditions.

To develop expressions for rate of current change, the derivatives of (1) and (5) are taken which gives

$$
\frac{d i}{d t}=\frac{v_{C F}(0) e^{-\alpha t}}{L}\left[\cos \left(\omega_{d} t\right)-\frac{\alpha}{\omega_{d}} \sin \left(\omega_{d} t\right)\right]
$$

and

$$
\frac{d i}{d t}=\frac{v_{C F}(0)}{L\left(s_{1}-s_{2}\right)}\left(s_{1} e^{s_{1} t}-s_{2} e^{s_{2} t}\right)
$$

respectively.

Expressions for the rate of change of voltage are found from the derivatives of (9) and (10), which are

$$
\frac{d v}{d t}=\frac{v_{C F}(0) \omega_{0}^{2}}{\omega_{d}} e^{-\alpha t} \sin \left(\omega_{d} t\right)
$$

and

$$
\frac{d v}{d t}=\frac{v_{C F}(0) \omega_{0}^{2}}{\left(s_{1}-s_{2}\right)}\left(e^{s_{1} t}-e^{s_{2} t}\right)
$$

respectively.

Finally the network impedance under fault conditions can be found from the division of the voltage expressions given in (9) and (10) by the equivalent current expressions presented in (1) and (5). In this manner, the underdamped response impedance can be expressed as

$$
Z(t)=-L \omega_{d} \cot \left(\omega_{d} t\right)-\frac{R}{2}
$$

and the overdamped impedance is equal to

$$
Z(t)=\frac{\left(\frac{e^{s_{1} t}}{s_{1}}-\frac{e^{s_{2} t}}{s_{2}}\right)}{C\left(e^{s_{1} t}-e^{s_{2} t}\right)} .
$$

Having derived these expressions it can be seen how the various circuit parameters shape the transient response. For example, $\alpha$ shows how the resistance and inductance parameters affect the exponential decay. $\omega_{d}$ illustrates how the combination of all circuit parameters can affect oscillatory frequency and hence the peak current time. Finally (6) shows how the damping and frequency terms equate to give the roots of the characteristic equation and hence establish the appropriate equations to use in the analysis.

The equations can be analyzed in detail to describe a number of aspects of the different fault responses. However, for the purposes of this paper, there are two key characteristics which the analysis reveals.

First, the equations illustrate the impact of damping on the network response, emphasizing the high sensitivity of the different responses to fault impedance. This is a result of the relatively short interconnecting cables (and hence small cable impedance) within microgrids. The effect of this is that the response is less dependent on location for impedance faults, making discrimination more difficult. This leads to the design of protection schemes based more on time based grading and less on magnitude based grading, which is undesirable for the intended application given the tight requirements on protection system operating time. Both of these characteristics are examined in further detail within [21].

One exception to this is the $d i / d t$ response under initial conditions, where a similarity exists for both under and overdamped fault conditions at the same fault location (that is, for variable impedance fault) [21]. A fault detection method is currently being developed by the authors based on this characteristic [22]. 
This approach would however be limited to the protection of a single line, and so could not replace network wide protection.

Second, a comparison of all of equations, with respect to damping, highlights that they display similar behavioral characteristics. This is an unsurprising finding given that they are all derived from the capacitive response. As such, any conclusions regarding the effectiveness of non-unit protection methods drawn on the basis of current based methods will be relevant for all potential non-unit measurands.

The following section will demonstrate the challenges in implementing overcurrent techniques to provide effective protection to the network illustrated in Fig. 1, looking specifically at the network's current and $i^{2} t$ responses to a range of fault conditions.

\section{B. Illustration of Detection Challenges Based on an All Overcurrent Protection Scheme}

To assess the capabilities of an overcurrent protection scheme to deliver the required levels of performance, this section looks at the coordination of pairs of upstream and parallel downstream devices, relating them to the previously derived operating requirements, and highlighting how these operating requirements differ depending on the connection of downstream devices. The merits of specific current-time graded protection schemes are not analyzed, as is perhaps more standard, as the authors believe the issues are more clearly demonstrated with a study of network response rather than detailed device characteristics. It is worth noting however that a relay operated on the extremely inverse current-time characteristic (designed for fast operating conditions) would behave in a similar manner to that a device operated on $i^{2} t$ [16]. Furthermore, [23] does discuss the potential issues in coordinating current-time characteristics for networks with large capacitive sources.

Whilst it is standard practice to coordinate protection device operation beginning with the furthest downstream device, the section instead first assesses the coordination of upstream devices because of the challenges associated with operating close to the capacitive source and the impact this has on downstream protection operation. These challenges are illustrated in the following sections.

1) Coordination of $P_{1}$ With $P_{2}$ and $P_{3}$ : To achieve good performance when coordinating $P_{1}$ with $P_{2}$ and $P_{3}$, the protection system must ensure that: any faults on line $P_{1}$ are quickly discriminated and cleared and $P_{1}$ remains stable for faults on downstream lines but provides backup in the event that $P_{2}$ or $P_{3}$ fail to operate.

As will be shown in later figures, the detection and discrimination of a low impedance fault at $F_{1}$ is reasonably straight forward given the excessive overcurrent produced compared to more distant faults. Therefore the objective for the protection system for close up faults is to operate sufficiently quickly to prevent damage at the point of fault and to components supplying fault current. Instead, the key coordination challenge in setting the overcurrent threshold at $P_{1}$ relates to the network fault response for higher impedance faults. To illustrate why this is the case, consider the plot shown in Fig. 2.

Fig. 2 illustrates the response of the network to 1 - and 500-m $\Omega$ faults at $F_{1}$, values which have been chosen to be
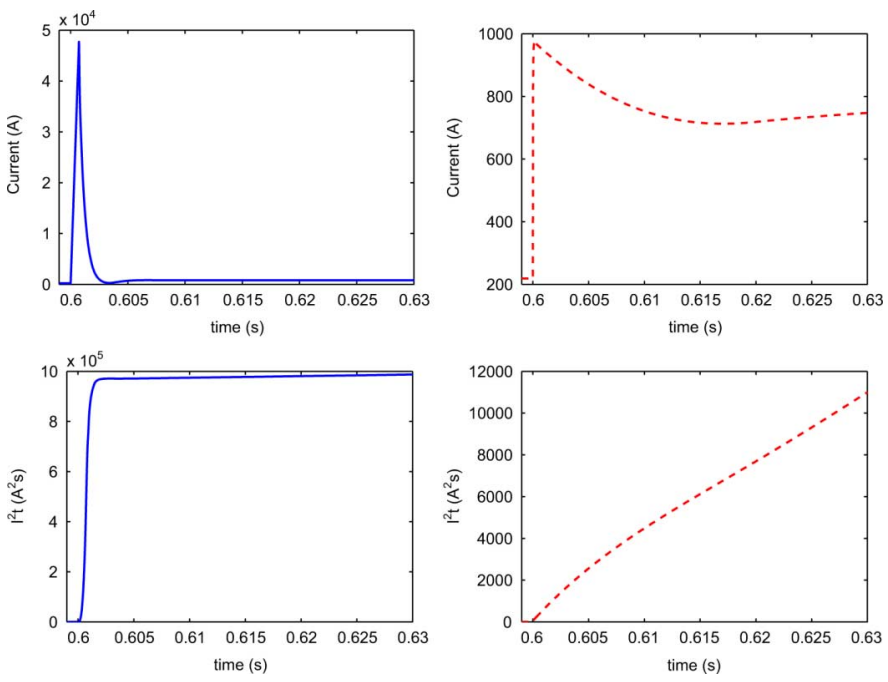

Fig. 2. Current (top) and $i^{2} t$ (bottom) response for $1-\mathrm{m} \Omega$ (left) and 500-m $\Omega$ (right) faults at $F_{1}$.

representative of low and high impedance fault conditions. It can be seen from Fig. 2 that for the two fault types, the peak fault current is vastly different, emphasizing the dominance of the fault impedance relative to the total fault path impedance. However in both cases the steady state output of the converter tends to the same level as the converter attempts to maintain output voltage to nominal levels. The magnitude of this steady state current will depend on either ac side fault level or converter rating (if the converter is capable of limiting current for dc faults). Whilst the network voltage will not decay to the same extent for impedance fault conditions (and potentially not reducing below the defined voltage threshold), the operating requirement relates to the converter's ability to supply this fault current.

This causes a problem in setting the overcurrent threshold for $P_{1}$. For example, if an initial threshold is set for $P_{1}$ as the $i^{2} t$ at the undervoltage threshold (set in the previous section as $0.9 \mathrm{~ms}$, at which point $i^{2} t$ equals $7.5 \times 10^{5} \mathrm{~A}^{2} \mathrm{~s}$ ), expanding the $i^{2} t$ plot for the $500-\mathrm{m} \Omega$ fault within Fig. 2 will show that it takes $1.18 \mathrm{~s}$ after fault inception to reach the same $\mathrm{A}^{2} \mathrm{~s}$ value. This would lead to the converter supplying fault current for longer than desired, and hence there is a requirement to lower this operating threshold from this initial level. However to maintain coordination with downstream devices, there is a limited degree to which this can be achieved. To assess the scope for the reduction, consider the current and $i^{2} t$ for $1 \mathrm{~m} \Omega$ fault at $F_{2}$ and $F_{3}$ shown in Fig. 3. Note that due to faults $F_{2}$ and $F_{3}$ being the same distance from the converter, and suitably low impedance, the responses to either fault is equivalent.

From a comparison of Fig. 3 and Table II it can be determined that the undervoltage threshold crossing at $2.2 \mathrm{~ms}$ corresponds to an $i^{2} t$ value of $6 \times 10^{4} \mathrm{~A}^{2} \mathrm{~s}$. Relating this value to the previous fault case, $6 \times 10^{4} \mathrm{~A}^{2} \mathrm{~s}$ is reached $0.16 \mathrm{~s}$ following the inception of fault $F_{1}-500 \mathrm{~m} \Omega$. Whilst this is perhaps longer than is desirable, it is reasonable to assume that the converter could supply current for this shorter time given the slower decay of dc side voltage. Therefore one protection setting option would be to reduce the threshold at $P_{1}$ to this level. However to maintain a 

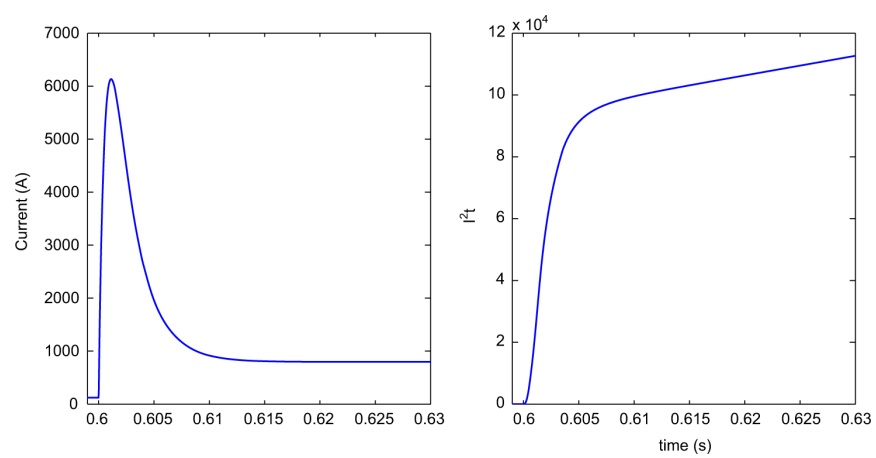

Fig. 3. Current (left) and $i^{2} t$ (right) response for 1-m $\Omega$ fault at $F_{2}$ and $F_{3}$.

TABLE III

Summary of Operating Threshold Times of $P_{1}, P_{2}$ AND $P_{3}$ FOR A FAULT AT $F_{2}$ OR $F_{3}$

\begin{tabular}{lllllll}
\hline Fault Location & $\begin{array}{l}t_{P_{1}} \\
\text { (initial) }\end{array}$ & $\begin{array}{l}t_{P_{2,3}} \\
\text { (initial) }\end{array}$ & $\begin{array}{l}t_{P_{1}} \\
\text { (revised) }\end{array}$ & $\begin{array}{l}t_{P_{2,3}} \\
\text { (revised) }\end{array}$ & $\begin{array}{l}\Delta t_{P_{1}-P_{2,3}} \\
\text { (initial) }\end{array}$ & $\begin{array}{l}\Delta t_{P_{1}}-P_{P_{2,3}} \\
\text { (revised) }\end{array}$ \\
\hline$F_{2,3}$ & $1.02 \mathrm{~s}$ & $2.2 \mathrm{~ms}$ & 2.2ms & $1.2 \mathrm{~ms}$ & $1.02 \mathrm{~s}$ & $1 \mathrm{~ms}$ \\
\hline
\end{tabular}

suitable time margin between the operating points of upstream and downstream protection (to enable device coordination), it is also necessary to reduce the thresholds of $P_{2}$ and $P_{3}$. This however brings its own problems given the need for $P_{3}$ to coordinate with further downstream devices and hence reducing the scope for threshold reduction. The necessity to reduce thresholds to achieve acceptable operating times does indicate that options to ride through the initial capacitive discharge, as suggested in [1] and [23], are limited.

To continue this example, consider the potential for circuit breaker coordination when reducing the threshold setting of $P_{2}$ and $P_{3}$ to $3 \times 10^{4} \mathrm{~A}^{2} \mathrm{~s}$ (half the original setting). Table III summarizes the times at which the thresholds will be reached for the initial and revised protection settings.

Table III highlights that whilst the initial protection settings were challenging to meet because of the short time frame, a sufficient time margin existed between upstream and downstream protection to ensure coordinated protection operation. However given the requirement to reduce the upstream $i^{2} t$ threshold to achieve reasonable operating times, the subsequent impact means that the time margin between device operations has reduced to a level such that on protection coordination is extremely difficult to achieve. This is in part due to the typical delay time between detection and circuit breaker operation, and this is discussed in more depth in later a section.

In order to increase the time margin between different device operations, there may be some scope for reduction in the threshold of $P_{2}$, albeit limited, given that it does not need to coordinate with further downstream devices. This is not the case for $P_{3}$, so further reduction in its threshold is not necessarily an option. To quantify this, the following sections investigate the impact of upstream device coordination on the response of downstream protection.

2) Coordination of $P_{3}$ With $P_{4}$ and $P_{5}$ : The potential for $P_{3}$ threshold reduction can be examined from analysis of downstream faults $F_{4,5}$. The initial threshold for $P_{4,5}$, derived from
TABLE IV

Summary of Operating Threshold Times of $P_{3}, P_{4}$, AND $P_{5}$ FOR A FAULT AT $F_{4}$ OR $F_{5}$

\begin{tabular}{lllllll}
\hline Fault Location & $\begin{array}{llll}t_{P_{3}} \\
\text { (initial) }\end{array}$ & $\begin{array}{l}t_{P_{4,5}} \\
\text { (initial) }\end{array}$ & $\begin{array}{l}t_{P_{3}} \\
\text { (revised) }\end{array}$ & $\begin{array}{l}t_{P_{4,5}} \\
\text { (revised) }\end{array}$ & $\begin{array}{l}\Delta t_{P_{3}-P_{4,5}} \\
\text { (initial) }\end{array}$ & $\begin{array}{l}\Delta t_{P_{3}-P_{4,5}} \\
\text { (revised) }\end{array}$ \\
\hline$F_{4,5}$ & $9.2 \mathrm{~ms}$ & $3.7 \mathrm{~ms}$ & $2.5 \mathrm{~ms}$ & $1.5 \mathrm{~ms}$ & $4.1 \mathrm{~ms}$ & $1 \mathrm{~ms}$ \\
\hline
\end{tabular}

TABLE V

Summary OF Operating Threshold Times of $P_{5}$ AND $P_{6}$ FOR A FAULT AT $F_{6}$

\begin{tabular}{lllllll}
\hline Fault Location & $\begin{array}{l}t_{P_{5}} \\
\text { (initial) }\end{array}$ & $\begin{array}{l}t_{P_{6}} \\
\text { (initial) }\end{array}$ & $\begin{array}{l}t_{P_{5}} \\
\text { (revised) }\end{array}$ & $\begin{array}{l}t_{P_{6}} \\
\text { (revised) }\end{array}$ & $\begin{array}{l}\Delta t_{P_{5}}-P_{6} \\
\text { (initial) }\end{array}$ & $\begin{array}{l}\Delta t_{P_{5}-P_{6}} \\
\text { (revised) }\end{array}$ \\
\hline$F_{4,5}$ & $9.4 \mathrm{~ms}$ & $5.3 \mathrm{~ms}$ & $2.3 \mathrm{~ms}$ & $1.4 \mathrm{~ms}$ & $4.1 \mathrm{~ms}$ & $0.9 \mathrm{~ms}$ \\
\hline
\end{tabular}

the undervoltage cut off, is $4.1 \times 10^{4} \mathrm{~A}^{2} \mathrm{~s}$. As this is greater than the revised threshold for $P_{3}$ in the previous section, there is a need to reduce this level. To maintain consistency with the previous section, the threshold for $P_{4,5}$ has been reduced to $1.5 \times 10^{4} \mathrm{~A}^{2} \mathrm{~S}$ (half that of $P_{3}$ ). A summary of the impact of this on required operating time and time margins is shown in Table IV.

Table IV highlights that the difference in required operating time for the initial undervoltage thresholds is already very tight and the impact of the reduced operating threshold compounds this problem, making the setting of devices extremely difficult. As with the previous case, given that the required operating time is already small, there is little scope for accelerating protection operation through threshold reduction. However for completeness, and to quantify challenges further downstream protection, the following section assesses the options for coordination of $P_{5}$ with $P_{6}$ and $P_{7}$.

3) Coordination of $P_{5}$ With $P_{6}$ and $P_{7}$ : In a similar manner to the previous section, the potential for device coordination is assessed through comparison of the initial and revised overcurrent thresholds. The initial $i^{2} t$ threshold for $P_{6}$ or $P_{7}$ was $3.13 \times 10^{4} \mathrm{~A}^{2} \mathrm{~s}$, which is again greater than revised upstream levels, and so in line with previous sections the $P_{6,7}$ it has been reduced to $0.75 \times 10^{4} \mathrm{~A}^{2} \mathrm{~s}$ (half of $P_{5}$ ). A summary of initial and revised operating times for a fault at $F_{6}$ is presented in Table $\mathrm{V}$.

Table $\mathrm{V}$ shows a similar trend to the previous section in terms of both required operating time and time difference between upstream and downstream devices. Therefore the device coordination challenges are similar to those reported previously.

\section{Discussion of Results}

The results presented in the previous sections have demonstrated the significant challenges which exist in the coordination of protection in compact de power systems, within the time constraints imposed by converter interfaces, using overcurrent based protection schemes.

In each scenario it was illustrated that the time margin between upstream and downstream protection operation was prohibitively small, creating a risk of upstream protection operation for downstream faults. This was in part due to the tight operating requirements from the network voltage response. However the need for reduction in the threshold of $P_{1}$ (to achieve reasonable operating times under impedance fault conditions) 
has a cascading effect on the downstream device settings and hence reduces operating margins. From this, it is worth noting in Table IV and V the time difference between the initial upstream and the revised downstream threshold is twice that of the difference between the two initial settings. This suggests that if the constraint of lowering the upstream threshold is removed, a greater opportunity for device coordination exists, provided operating requirements are met.

It is also worth considering how the difference in required operating time compares to that of the physical operating speeds of circuit breakers. Previous work by the authors [6] highlights that the requirement for fast acting protection can limit the range of protection devices which can be employed in dc microgrid networks. For example, the operating time of dc electro-mechanical circuit breakers (EMCBs) can be from around $3 \mathrm{~ms}$ [24], which exceeds the time difference in the scenarios described in the previous section, meaning coordination is not necessarily possible using the methods presented. Solid state and hybrid circuit breaker technologies offer a potential alternative to EMCBs, as these breakers can operate extremely quickly after the detection of a fault, however there are greater limitations on the operating voltage and current levels of these devices than for EMCBs.

It must also be appreciated that dc current breaking cannot be achieved instantaneously and there is a finite time when current is driven to zero. During this period current will continue to flow through upstream devices [25] and this could, depending on network conditions, cause an upstream device to operate before the fault is fully cleared.

Acknowledging these shortfalls, it can be concluded that the non-unit methods analyzed are unlikely to be able to achieve the required levels of protection discrimination for the derived operating requirements. Within future dc networks it is likely that a higher level of fault discrimination will be desirable, particularly if $\mathrm{dc}$ is to be proven a viable alternative to ac distribution. For these future networks, it has been demonstrated that for this to be achieved, more robust protection approaches are required. The following section investigates the potential for unit protection schemes to provide this required protection performance and proposes commercial off the shelf (COTS) based solutions for the key challenges identified. This analysis subsequently enables the development of a protection framework for dc microgrid applications.

\section{Application of Unit Protection}

Unit protection protects a clearly bounded zone of the power system and will not operate for faults external to this zone. In contrast to non-unit schemes, it does not provide backup to adjacent elements of the system [26] hence non-unit protection is often deployed alongside unit protection to provide the necessary backup functionality. A common form of unit protection is current differential protection, which operates by comparing all currents' magnitudes and/or relative directions at the boundaries of a specified element within a network [26].

The nature of the current differential protection method is such that it is far less susceptible to the effects of variable fault levels and impedances than non-unit methods [27], facilitating effective protection coordination in the network. However, the major challenge which remains for the implementation of a differential protection scheme within dc microgrids is the capability to achieve fault detection within the time frame described in previous sections.

In addition to the technical constraints, the economics of the protection system implementation must also be considered [1]. The requirements for device communication means the installation costs of a current differential scheme can be substantial compared to an overcurrent scheme, particularly at the distribution level, although this might be somewhat reduced for microgrid systems due to their relatively small geographical area. However, given the likely increase in the amount of sensor and communication infrastructure within distribution networks [9] as smart grid and microgrid [8] concepts develop, much of the required infrastructure may already be in place. The deployment of this advanced infrastructure therefore provides an opportunity to expand the use of unit (communication-based) protection schemes within microgrids.

To explore this opportunity, this section considers two aspects to the implementation of current differential protection. The first aims to quantify these implementation challenges and the second considers the impact of unit protection implementation on overall protection. These aspects are described in the following subsections.

\section{A. Challenges and Proposed Solutions for the Implementation of Fast Acting Unit Protection Schemes}

There are two main challenges for the implementation of unit protection within the highly transient environment described in the previous sections. The first is, can currents be compared and fault location determined within the required time frame? The second is, can the current measurements at different points in the network be accurately synchronized to ensure correct protection system operation? These issues are discussed in the following sections.

1) Challenges in Meeting the Operating Requirement: Current differential protection applied in ac systems typically has a target operation time of 1-2 cycles, which often represents an operation time of $>20 \mathrm{~ms}$ [28], [29]. In comparison, the operating times derived in Section II are much shorter and hence alternative implementation methods must be deployed in order to meet these operating times.

One factor which prevents the reduction in operating time of an ac current differential system is the requirement for individual phase current measurement and phasor comparison [26]. This requirement does not exist for dc implementation, where only current magnitudes need to be compared. Furthermore, as dc current will be measured using a current transducer (such as a Hall Effect device) rather than via a current transformer, the measurement will be in the form of voltage which facilitates easier integration with digital processing devices. As [6] suggests, the sampling rates available on modern microprocessors are such that numerous current samples could be summed and a fault detected well within the derived operating requirements. Therefore the dominant factor for fault detection within dc networks is the communication delay between different devices. A dc current differential scheme for the network described within this paper must therefore be supported by a high bandwidth 


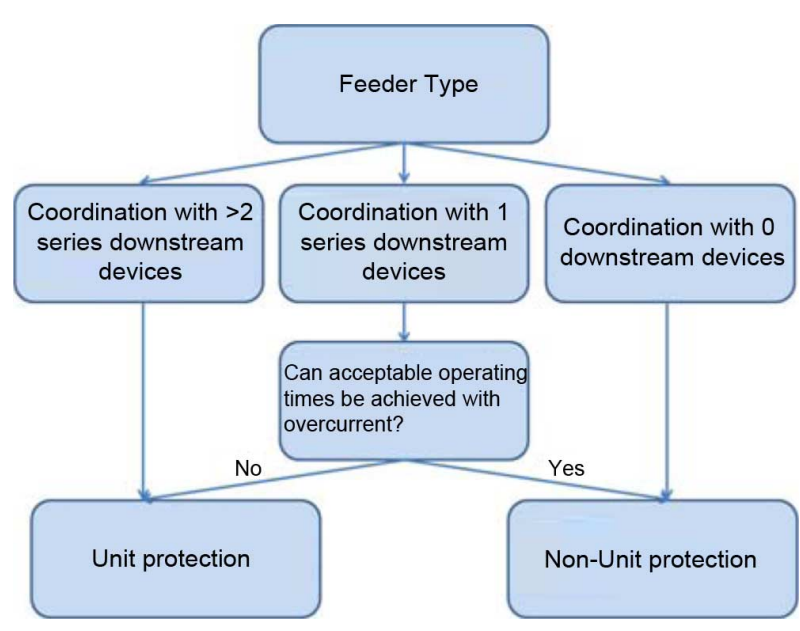

Fig. 4. Protection scheme approach decision tree.

communications system in order to operate effectively within the derived operating times. Exact specification of these requirements is an area of future work.

2) Challenges in the Implementation of Unit Systems When Operating Under High Rate of Change Fault Conditions: To avoid the occurrence of errors in the current differential sum, and hence ensure that the scheme performs correctly, current measurements must be synchronized in time [26]. There are a number of potential sources of poor time synchronization. These include timing errors between communicating devices and nonsynchronous current sampling. Given the high rate of current change in the fault response considered within this paper (initially $\approx 17 \mathrm{A \mu} \mu \mathrm{s}^{-1}$ for a fault at $F_{2}$ for example), accurate device synchronization becomes even more important to ensure the protection scheme does not mal-operate.

To minimize the impact of this on the protection system operation in practice, protection relays often communicate both current and time information to ensure current measurements are compared in the same point in time. Synchronization using this approach can be most effectively achieved where devices are synchronized through GPS time stamping [28], where devices are synchronized with an accuracy of $\pm 1 \mu \mathrm{s}$. It is anticipated that this degree of accuracy would be sufficient however if the time difference was to increase then there is a possibility of large errors occurring within the differential sum.

\section{B. Protection System Design Framework for DC Microgrid Applications}

In this section, a protection system design framework is proposed which provides clear design guidance with which to achieve fast and effective protection system operation, whilst seeking to minimize installation costs, against a set of very strict operating requirements. It achieves this by proposing the use of unit protection upstream within a network in order to ease the constraints on downstream non-unit protection. Fig. 4 provides a pictorial overview of the design framework.

The proposed framework provides guidance on the required protection approach for a feeder based on its position within the network and the surrounding elements of this network. In this manner, unit protection methods (with non-unit backup) are recommended for feeders where coordination with downstream devices is likely to be difficult within an acceptable time frame, but non-unit protection is recommended for all other cases in order to balance required system performance against installation cost. This framework is applicable to dc microgrids of various configurations.

To illustrate the underlying philosophy of this scheme, consider the example microgrid network illustrated in Fig. 1. If a current differential scheme were to be applied between the supply converter output and the first parallel connection point (prior to $P_{2}$ and $P_{3}$ ) in place of an overcurrent scheme, it would serve to remove the constraint of reducing the $P_{1}$ threshold to achieve acceptable operating times under impedance fault conditions. This could be achieved as the unit protection zone would be insensitive to external faults and hence not operate even with high current throughput. The subsequent effect of this would be to enable the remainder of the protection settings within the network to return to the initial values derived from the time of undervoltage, increasing the time margin between the operation of different devices. However this still leaves very tight operating time requirements, particularly where devices have to coordinate with other downstream protection devices.

Additionally, Section III-B shows that the time margin between adjacent devices from $P_{3}$ onwards is similar, and this is due to the uniform fault separation and cable parameters within the network. To adhere to requirement of operating protection prior to a network undervoltage, it has been shown that the only means of increasing this time margin is to decrease the downstream threshold. This is possible between $P_{3}$ and $P_{4}$, however due to the connection of additional parallel loads downstream for $P_{5}$, potential reduction in the overcurrent threshold at $P_{5}$ is limited. The application of unit protection at each of these parallel connection points would not only ensure accurate fault detection for internal zone faults but also that there is sufficient time available for the operation of protection devices for load connection points. Protection of these parts of the network could be achieved through the use of simpler non-unit techniques.

\section{CONCLUSION}

The development of effective protection system solutions is a critical step in the development of high performance dc microgrid systems. The key contribution of this paper is to identify the means with which to achieve fast and effective protection system operation, whilst seeking to minimize installation costs, against a set of very strict operating requirements. The paper has demonstrated the limitations of non-unit protection methods to achieve effective fault discrimination within derived operating times and the use of current differential protection is introduced as a potential solution. However this has implications for system cost and complexity. Following the analysis of these protection methods, the potential roles of unit and non-unit protection methods are defined within the example microgrid network. Extrapolating this analysis, a design framework is proposed for $\mathrm{dc}$ microgrid systems which provides a means of optimizing protection scheme design to achieve required fault discrimination and operating speed in an economic way. Whilst of simple appearance, the clarity provided by this framework is only possible as a result of the underpinning analysis. 


\section{ACKNOWLEDGMENT}

This work has been carried out as part of the Rolls-Royce UTC program.

\section{REFERENCES}

[1] R. Cuzner and G. Venkataramanan, "The status of DC micro-grid protection," in Proc. IEEE Ind. Appl. Soc. Annu. Meeting (IAS), Oct. 2008, pp. 1-8.

[2] K. George, EPRI Solutions, Inc., "DC power production, delivery and utilization: An EPRI white paper," 2006.

[3] K. Shenai and K. Shah, "Smart DC micro-grid for efficient utilization of distributed renewable energy," presented at the IEEE Energytech, Cleveland, OH, May 2011.

[4] D. Salomonsson, L. Soder, and A. Sannino, "Protection of low-voltage DC microgrids," IEEE Trans. Power Del., vol. 24, no. 3, pp. 1045-1053, Jul. 2009.

[5] T. Kaipia, P. Salonen, J. Lassila, and J. Partanen, "Application of low voltage DC-distribution system-A techno-economical study," presented at the 19th Int. Conf. Elect. Distrib., Vienna, Austria, May 2007.

[6] S. Fletcher, P. Norman, S. Galloway, and G. Burt, "Determination of protection system requirements for DC unmanned aerial vehicle electrical power networks for enhanced capability and survivability," IET Elect. Syst. Transp., vol. 1, no. 4, pp. 137-147, 2011.

[7] M. E. Baran and N. R. Mahajan, "Overcurrent protection on voltagesource-converter-based multiterminal dc distribution systems," IEEE Trans. Power Del., vol. 22, no. 1, pp. 406-412, Jan. 2007.

[8] G. Venkataramanan and C. Marnay, "A larger role for microgrids," IEEE Power Energy Mag., vol. 6, no. 3, pp. 78-82, May-Jun. 2008.

[9] V. Sood, D. Fischer, J. Eklund, and T. Brown, "Developing a communication infrastructure for the smart grid," in Proc. IEEE Elect. Power Energy Conf. (EPEC), Oct. 2009, pp. 1-7.

[10] Short-Circuit Currents in DC Auxiliary Installations in Power Plants and sSubstations - Part 2: Calculation of eEffects, IEC 61660-2:1997, International Electrotechnical Commission, 1997.

[11] J. Yang, J. Fletcher, and J. O'Reilly, "Multiterminal dc wind farm collection grid internal fault analysis and protection design," IEEE Trans. Power Del., vol. 25, no. 4, pp. 2308-2318, Oct. 2010.

[12] L. Tang and B.-T. Ooi, "Locating and isolating DC faults in multiterminal DC systems," IEEE Trans. Power Del., vol. 22, no. 3, pp. 1877-1884, Jul. 2007.

[13] J. Candelaria and J.-D. Park, "Vsc-hvdc system protection: A review of current methods," in Proc. IEEE/PES Power Syst. Conf. Expo. (PSCE), Mar. 2011, pp. 1-7.

[14] National Grid Electricity Transmission plc, "The grid code," no. 4, revision 1, May 16, 2010 [Online]. Available: http://www.nationalgrid.com/uk/Electricity/Codes/gridcode/

[15] K. I. Jennett, C. D. Booth, and L. Martin, "Analysis of the sympathetic tripping problem for networks with high penetrations of distributed generation," presented at the Int. Conf. Adv. Power Syst. Autom. Prot., Beijing, China, Oct. 2011.

[16] "Overcurrent protection for phase and earth faults," in Network Protection and Automation Guide ALSTOM, 2011, ch. 9 [Online]. Available: www.alstom.com/grid

[17] R. M. Tumilty, M. Brucoli, G. M. Burt, and T. C. Green, "Approaches to network protection for inverter dominated electrical distribution systems," in Proc. IET 3rd Int. Conf. Power Electron., Mach. Drives, Mar. 2006, pp. 622-626.

[18] E. Cinieri, A. Fumi, V. Salvatori, and C. Spalvieri, "A new high-speed digital relay protection of the 3-kvdc electric railway lines," IEEE Trans. Power Del., vol. 22, no. 4, pp. 2262-2270, Oct. 2007.

[19] S. Foster, L. Xu, and B. Fox, "Control of an LCC HVDC system for connecting large offshore wind farms with special consideration of grid fault," in IEEE Power Energy Soc. Gen. Meeting, Jul. 2008, pp. 1-8.

[20] "Distance protection," in Network Protection and Automation Guide ALSTOM, 11 [Online]. Available: www.alstom.com/grid

[21] S. Fletcher, P. Norman, S. Galloway, and G. Burt, "Analysis of the effectiveness of non-unit protection methods within dc microgrids," presented at the IET Conf. Renew. Power Gen., Edinburgh, U.K., Sep. 2011.

[22] S. D. A. Fletcher, P. J. Norman, S. J. Galloway, and J. E. Hill, "Protection system for an electrical power network," U.K. Patent application GB1102031.0, Feb. 2011
[23] R. Cuzner, D. MacFarlin, D. Clinger, M. Rumney, and G. Castles, "Circuit breaker protection considerations in power converter-fed DC systems," in IEEE Elect. Ship Tech. Symp., Apr. 2009, pp. 360-367.

[24] "Secheron high-speed dc circuit-breaker for rolling stock type UR26," 2011. [Online]. Available: http://www.secheron.com

[25] S. D. A. Fletcher, P. Norman, S. Galloway, and G. Burt, "Solid state circuit breakers enabling optimised protection of dc aircraft power systems," presented at the 14th Eur. Conf. Power Electron. Appl. (EPE), Birmingham, U.K., Sep. 2011

[26] "Unit protection of feeders," in Network Protection and Automation Guide ALSTOM, 10 [Online]. Available: www.alstom.com/grid

[27] P. Karlsson and J. Svensson, "Fault detection and clearance in DC distributed systems," presented at the Nordic Worskshop Power Ind. Electron., Stockholm, Sweden, Aug. 2002.

[28] N. Villamagna and P. Crossley, "A symmetrical component-based GPS signal failure-detection algorithm for use in feeder current differential protection," IEEE Trans. Power Del., vol. 23, no. 4, pp. 1821-1828, Oct. 2008.

[29] SEL-inc, "SEL-311L line current differential protection and automation system," 2011. [Online]. Available: http://www.selinc.com

Steven D. A. Fletcher received the B.Eng. (Hons) degree in electrical and electronic engineering from the University of Strathclyde, Glasgow, U.K., in 2007, where he is currently working toward the Ph.D. degree.

$\mathrm{He}$ is currently a Research Assistant with the Institute for Energy and Environment, University of Strathclyde. His research interests include the transient analysis and protection of dc systems for microgrid, marine, and aerospace applications.

Patrick J. Norman received the B.Eng. (Hons) degree in electrical and mechanical engineering and $\mathrm{Ph} . \mathrm{D}$. degree in electrical engineering from the University of Strathclyde, Glasgow, U.K.

$\mathrm{He}$ is a Full-Time Research Staff Member with the Institute for Energy and Environment, University of Strathclyde. He has published over 35 peer reviewed journal and conference publications. His current research interests lie in the modeling and simulation, design, control, protection of aircraft secondary power offtake and distribution systems, and microgrid and shipboard power systems.

Stuart J. Galloway received the Bachelors degree in mathematical sciences from the University of Paisley, Paisley, U.K., in 1992, and the M.Sc. degree in nonlinear modeling and Ph.D. degree in numerical analysis from the University of Edinburgh, Edinburgh, U.K., in 1993 and 1998, respectively.

$\mathrm{He}$ is currently a Senior Lecturer with the Institute for Energy and Environment, University of Strathclyde. Since 1998, he has been researching optimization problems in power systems, electricity markets, and novel electrical architectures relating to aero and marine electrical systems.

Paul Crolla (M'06) received the M.Sc. degree in physics from the University of Strathclyde, Glasgow, U.K., in 2005.

He is currently a Research Assistant with the Protection, Automation and Control Team, Institute for Energy and Environment, University of Strathclyde. His main research interests lie in the development of power hardware-in-theloop applications and power systems laboratory demonstration.

Graeme M. Burt (M'95) received the B.Eng. degree in electrical and electronic engineering and the Ph.D. degree from the University of Strathclyde, Glasgow, U.K., in 1988 and 1992, respectively, following research into fault diagnostic techniques for power networks.

He is currently a Director of the Institute for Energy and Environment, University of Strathclyde, where he also directs the University Technology Centre in Electrical Power Systems sponsored by Rolls-Royce. He is a Professor of electrical power engineering, and has particular research interests in the areas of integration of distributed generation, power system modeling and real-time simulation, power system protection and control, microgrids, and more-electric systems. 